\title{
Alantolactone induces gastric cancer BGC-823 cell apoptosis by regulating reactive oxygen species generation and the AKT signaling pathway
}

\author{
$\mathrm{XIN}_{\mathrm{ZHANG}^{1}}$ and HONG-MING ZHANG ${ }^{2}$ \\ ${ }^{1}$ Department of Gastroenterology, People's Hospital, Chongqing 401120; ${ }^{2}$ Department of Blood Transfusion, \\ General Hospital of Xinjiang Military Area Command for The People's Liberation Army, \\ Urumqi, Xinjiang Uygur Autonomous Region 830000, P.R. China
}

Received December 22, 2017; Accepted January 21, 2019

DOI: $10.3892 / 01.2019 .10172$

\begin{abstract}
Alantolactone (ALT), a natural sesquiterpene lactone, has been suggested to exert anti-cancer activities in various cancer cell lines. However, the effects and mechanisms of action of ALT in human gastric cancer remains to be elucidated. In the present study, the effects of ALT on BGC-823 cells were examined and the underlying molecular mechanisms associated with these effects were investigated. Cell viability was detected by using an MTT assay. Cell cycle, cell apoptosis and the level of reactive oxygen species (ROS) were assessed by flow cytometry, and the expression levels of proteins of interest were analyzed by western blot assay. The results demonstrated that ALT triggered apoptosis and induced G0/G1 phase arrest in a dose-dependent manner. Furthermore, the expression level of the anti-apoptosis protein Bcl-2 was downregulated, and expression of the pro-apoptosis proteins Bax and cleaved PARP were significantly upregulated. The cell cycle-associated proteins cyclin-dependent kinase inhibitor 1 and cyclin-dependent kinase inhibitor 1B were also increased, while cyclin D1 was deceased. In addition, ALT induced apoptosis via the inhibition of RAC-alpha serine/threonine-protein kinase (AKT) signaling and ROS generation, which was effectively inhibited by the ROS scavenger, $\mathrm{N}$-acetyl cysteine. Therefore, the results from the present study indicated that the ROS-mediated inhibition of the AKT signaling pathway serves an important role in ALT-induced apoptosis in BGC-823 cells. In conclusion, the results demonstrated that ALT exerted significant anti-cancer effects against gastric cancer cells in vitro.
\end{abstract}

Correspondence to: Dr Xin Zhang, Department of Gastroenterology, People's Hospital, 62 Jianshe Road, Shuanglonghu Street, Yubei, Chongqing 401120, P.R. China

E-mail: zx2608tmmu@aliyun.com

Key words: alantolactone, BGC-823 cells, apoptosis, reactive oxygen species, protein kinase B serine/threonine kinase signaling pathway

\section{Introduction}

Gastric carcinoma is currently the third most common cause of cancer mortality worldwide; $50 \%$ of all gastric carcinomas occur in Eastern Asia, and it is particularly common in China (1,2). At present, the effective treatment strategies for gastric carcinoma include surgery, radiotherapy, chemotherapy and targeted therapy $(3,4)$. Surgical resection followed by adjuvant chemotherapy remains the most effective therapeutic option. Unfortunately, relapse and metastasis of gastric tumors (5), and resistance to chemotherapy are common. Therefore, novel therapeutic agents for gastric carcinoma therapy are urgently required.

Increasing attention has been paid to the application of natural products for chemopreventative cancer therapy. Terpenoids are phytochemicals traditionally used for medicinal purposes. Preclinical studies have demonstrated that terpenoids present in plant foods or isolated from medicinal plants, including germacrone from Rhizoma Curcuma or lactucopicrin from Cichorium intybus $L$, exhibit significant anti-cancer effects against various types of cancer cell in vivo and in vitro (6-8). Alantolactone (ALT), a sesquiterpene lactone compound isolated from Inula helenium, has exhibited multiple biological properties, including anti-bacterial, anti-inflammatory and anti-cancer activities (9). Notably, ALT was suggested to exhibit potential anti-cancer activity against various types of cancer, including human colorectal cancer (10), liver cancer $(11,12)$, leukemia (13), breast cancer $(14)$, lung cancer $(15,16)$ and cervical cancer $(17,18)$. ALT may inhibit breast cancer growth via anti-angiogenic activity by inhibiting vascular endothelial growth factor receptor 2 and RAC-alpha serine/threonine-protein kinase B (AKT) signaling (19). In human cervical cancer cells, ALT induces apoptosis via generation of reactive oxygen species (ROS) and inhibition of the B-cell lymphoma 2 (Bcl-2)/Bcl-2 associated $\mathrm{X}$ apoptosis regulator (Bax) signaling pathway (17). In SK-MES-1 lung squamous cancer SK-MES-1 cells, ALT may trigger apoptosis and induce cell cycle G1/G0 phase arrest. Furthermore, ALT may enhance the chemosensitivity of A549 cells to doxorubicin via ROS-mediated apoptosis (20). However, the exact mechanism underlying the anti-cancer 
activity of ALT in human gastric cancer cells remains to be elucidated.

The present study aimed to elucidate the anti-cancer effects and associated molecular mechanisms of ALT in BGC-823 cells, and to evaluate the potential of ALT for its application as a novel naturally-derived agent for the treatment of gastric cancer.

\section{Materials and methods}

Reagents. ALT, N-acetyl cysteine (NAC) and MTT were purchased from Sigma-Aldrich; Merck KGaA (Darmstadt, Germany). Primary antibodies to detect cyclin D1 (cat. no., 2978), cyclin-dependent kinase inhibitor 1 (p21; cat. no., 2947), cyclin-dependent kinase inhibitor 1B (p27; cat. no., 3686), Bax (cat. no., 5023), Bcl-2 (cat. no., 15071), poly (adenosine 5'diphosphate-ribose) polymerase (PARP; cat. no., 5625), phosphorylated (p)-AKT (cat. no., 4060), AKT (cat. no., 9272) and GAPDH (cat. no., 5174) were obtained from Cell Signaling Technology, Inc. (Danvers, MA, USA). The Annexin V-fluorescein isothiocyanate (FITC)/propidium iodide (PI) apoptosis detection kit and cell cycle detection kit were purchased from Nanjing KeyGen Biotech Co. Ltd. (Nanjing, China). The ROS assay kit was purchased from Nanjing Jiancheng Bioengineering Institute (Nanjing, China). RPMI-1640 medium and fetal bovine serum (FBS) were purchased from Gibco; Thermo Fisher Scientific, Inc. (Waltham, MA, USA).

Cell culture. The BGC-823 human gastric cancer cell line was purchased from The Cell Bank of Type Culture Collection of the Chinese Academy of Sciences (Shanghai, China). The cells were cultured in RPMI-1640 medium supplemented with $10 \%$ FBS, $100 \mathrm{U} / \mathrm{ml}$ penicillin and $100 \mu \mathrm{g} / \mathrm{ml}$ streptomycin at $37^{\circ} \mathrm{C}$ with $5 \% \mathrm{CO}_{2}$.

MTT cytotoxicity assay. Cell viability was measured using an MTT assay as described previously (21). The BGC-823 cells were seeded into 96-well plates at a density of $1 \times 10^{5}$ cells/well and treated following culture for $24 \mathrm{~h}$. The BGC-823 cells were treated with $\operatorname{ALT}(0,10,20,40,60,80$ or $100 \mu \mathrm{M})$, and incubated for an additional $24 \mathrm{~h}$. In total, $0.5 \mathrm{mg} / \mathrm{ml}$ MTT solution was then added to the medium, and the cells were incubated for an additional $4 \mathrm{~h}$ at $37^{\circ} \mathrm{C}$. The culture was then removed, and dimethyl sulfoxide ( $150 \mu \mathrm{l} /$ well) was added to dissolve the solid residue. The absorbance at $490 \mathrm{~nm}$ (A490) was determined using an ELISA microplate reader, and all experiments were performed at least 3 times. Percentage of cell viability was calculated as follows: Cell viability $(\%)=(\mathrm{A} 490$ sample A490 blank)/(A490 control-A490 blank) x100.

Cell cycle assay. Cell cycle arrest was detected using a cell cycle detection kit according to manufacturer's protocol (Nanjing KeyGen Biotech Co., Ltd.). Briefly, the BGC-823 cells were seeded at $1 \times 10^{6}$ cells/well in 6 -well plates and treated with different ALT concentrations $(0,10,20,40$ or $60 \mu \mathrm{M})$ for $24 \mathrm{~h}$. The BGC-823 cells were fixed with $75 \%$ ethanol at $4^{\circ} \mathrm{C}$ overnight. PI was then used to stain the DNA of the samples for $15 \mathrm{~min}$ at $25^{\circ} \mathrm{C}$ in the dark, and flow cytometry analysis was performed using a
FACSCalibur flow cytometer (BD Biosciences, Franklin Lakes, NJ, USA) was used to determine the cell cycle and analyzed by Modfit LT 3.0 software (Verity Software House, Topsham, ME, USA). All experiments were performed at least three times.

Assessment of apoptosis. Cell apoptosis was assessed using an Annexin V-FITC/PI kit according to manufacturer's protocol (Nanjing KeyGen Biotech Co., Ltd.) and the apoptotic rate was analyzed by flow cytometry on a FACSCalibur ${ }^{\mathrm{TM}}$ flow cytometer (BD Biosciences). BGC-823 cells were seeded at $1 \times 10^{6}$ cells/well in 6-well plates and treated different ALT concentrations $(0,10,20,40$ or $60 \mu \mathrm{M})$ for $24 \mathrm{~h}$. Subsequently, cells were stained with Annexin V-FITC and PI in binding buffer for $15 \mathrm{~min}$ at $25^{\circ} \mathrm{C}$ in the dark. According to manufacturer's protocol, $5 \mu \mathrm{l}$ Annexin V-FITC and $5 \mu \mathrm{l}$ PI were added per sample. The apoptotic cells were then detected on a FACSCalibur ${ }^{\mathrm{TM}}$ flow cytometer (BD Biosciences) and analyzed using FlowJo 7.6 software (FlowJo LLC, Ashland, OR, USA). A total of three independent experiments were performed.

ROS detection. ROS generation was measured using a ROS assay kit according to the manufacturer's protocol (Nanjing Jiancheng Bioengineering Institute) and the ROS levels were analyzed by flow cytometry on a FACSCalibur ${ }^{\mathrm{TM}}$ flow cytometer (BD Biosciences). Briefly, BGC-823 cells were treated with the indicated concentrations $(0,10,20,40$ and $60 \mu \mathrm{M})$ of ALT for $6 \mathrm{~h}$. In addition, BGC-823 cells were pre-treated with $10 \mathrm{mM}$ NAC for $1 \mathrm{~h}$, and with $40 \mu \mathrm{M}$ ALT for $6 \mathrm{~h}$. The cells were then incubated with $30 \mu \mathrm{M}$ 2',7'-dichlorodihydrofluorescein diacetate at $37^{\circ} \mathrm{C}$ for $30 \mathrm{~min}$. The cells were analyzed using a FACSCalibur flow cytometer (BD Biosciences). The results were analyzed with FlowJo 7.6 software (FlowJo LLC) and all experiments were performed at least three times.

Western blot analysis. Cells were lysed using radioimmunoprecipitation assay (Beyotime Institute of Biotechnology, Haimen, China) on ice for $30 \mathrm{~min}$. The proteins were quantified with Bicinchoninic Acid Protein Assay kit (Thermo Fisher Scientific, Inc.). Proteins $(40 \mu \mathrm{g})$ were separated by 6-12\% SDS-PAGE and then transferred onto nitrocellulose membranes (Pall Life Sciences, Port Washington, NY, USA) and blocked with 5\% non-fat milk in TBS-containing $0.05 \%$ Tween 20 for $2 \mathrm{~h}$ at room temperature. The membranes were incubated with anti-cyclin D1 (1:1,000 dilution), anti-p21 (1:1,000 dilution), anti-p27 (1:1,000 dilution), anti-Bax (1:1,000 dilution), anti-Bcl-2 (1:1,000 dilution), anti-p-AKT (1:1,000 dilution), anti-AKT (1:1,000 dilution), anti-cleaved PARP and anti-GAPDH (1:3,000 dilution) antibodies at $4^{\circ} \mathrm{C}$ overnight. Membranes were then incubated with horseradish peroxidase (HRP)-conjugated anti-mouse immunoglobulin (Ig)G (cat. no. 7076; 1:25,000) and HRP-conjugated anti-rabbit IgG (cat. no. 7074; 1:20,000; both Cell Signaling Technology, Inc.) secondary antibodies for $2 \mathrm{~h}$ at $25^{\circ} \mathrm{C}$. Visualization was performed using a SuperSignal West Pico chemiluminescent substrate (Pierce; Thermo Fisher Scientific, Inc.).

Statistical analysis. Data are presented as the mean \pm standard deviation. Differences between groups were determined by 
one-way analysis of variance followed by Dunnett's or Tukey's post-hoc tests. The analyses were performed using SPSS v.19 statistical software (IBM Corp., Armonk, NY, USA). $\mathrm{P}<0.05$ was considered to indicate a statistically significant difference.

\section{Results}

ALT inhibits cell proliferation in BGC-823 cells. The effect of ALT on cell proliferation was determined using an MTT assay. The cytotoxicity of ALT was evaluated by treating BGC-823 cells with different concentrations (0, 10, 20, 40, 60,80 and $100 \mu \mathrm{M}$ ) for $24 \mathrm{~h}$. As demonstrated in Fig. 1, ALT significantly inhibited the viability of BGC-823 cells in a concentration-dependent manner, exhibiting significant differences compared with the control group. These results indicated that ALT significantly suppressed the growth of BGC-823 human gastric cancer cells.

ALT induces cell cycle arrest at the G0/G1 phase in $B G C-823$ cells. Inhibition of cell proliferation via anti-tumor drugs is often accompanied by changes in cell cycle progression $(22,23)$. In the present study, flow cytometry was used to analyze the distribution of the cell cycle in BGC-823 cells following treatment with ALT $(20-60 \mu \mathrm{M})$ for $24 \mathrm{~h}$. G0/G1 phase arrest was observed in BGC-823 cells exposed to ALT compared with the control group. As demonstrated in Fig. 2A, ALT induced $\mathrm{G} 0 / \mathrm{G} 1$ phase arrest of BGC-823 cells in a dose-dependent manner. The percentage of cells in the G0/G1 phase was $57.50 \pm 0.10,66.57 .50 \pm 0.05,71.24 \pm 0.88,72.31 \pm 0.17$ and $81.97 \pm 0.53 \%$ in the $0,10,20,40$ and $60 \mu \mathrm{M}$ groups, respectively (Fig. 2A). To gain insight into the mechanism of G0/G1 phase arrest, the expression levels of cyclin D1, p21 and p27, which regulate $\mathrm{G} 1$ to $\mathrm{S}$ phase progression, were detected. As presented in Fig. 2B, the level of cyclin D1 was decreased, and levels of p21 and p27 were significantly increased following stimulation with ALT. These results indicated that ALT induces G0/G1 phase arrest via downregulation of cyclin D1 expression and upregulation of p21 and p27 expression in BGC-823 cells.

ALT induces apoptosis in BGC-823 cells. Anti-tumor drugs often execute cytotoxic effects via the induction of apoptosis $(24,25)$. The effect of ALT on BGC-823 cell apoptosis was evaluated using Annexin V-FITC/PI double staining and flow cytometry. The percentage of apoptotic cells was $1.15 \pm 0.01,22.69 \pm 0.13,25.10 \pm 0.14,39.53 \pm 0.37$ and $50.93 \pm 0.29 \%$ in the $0,10,2,40$ and $60 \mu \mathrm{M}$ groups, respectively, after $24 \mathrm{~h}$ (Fig. 3A). The data suggested that ALT significantly induced apoptosis in a dose-dependent manner. To reveal the molecular mechanism involved in ALT-induced apoptosis, the levels of anti-apoptosis protein Bcl-2 and pro-apoptosis proteins Bax and cleaved PARP were detected by western blot analysis. When BGC- 823 cells were treated with $40 \mu \mathrm{M}$ ALT, Bax and cleaved PARP protein levels were increased in a time-dependent manner, whereas Bcl-2 levels were decreased in a time-dependent manner (Fig. 3B). These results suggested that ALT may trigger apoptosis in BGC-823 cells partially via a mitochondrial-dependent pathway.

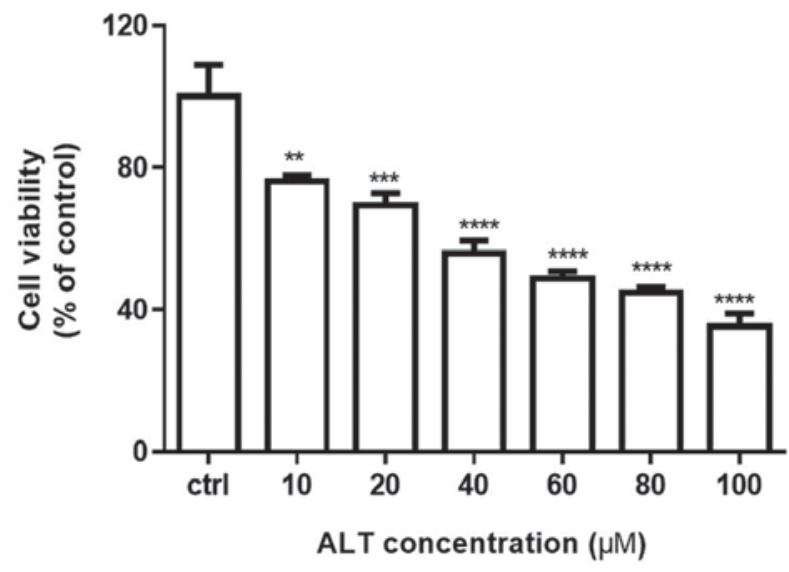

Figure 1. Effects of ALT on the viability of BGC-823 cancer cells. BGC-823 cells were incubated with different concentrations of ALT $(0-100 \mu \mathrm{M})$ for $24 \mathrm{~h}$. All data are expressed as means \pm standard deviation from 3 independent experiments. ${ }^{* *} \mathrm{P}<0.01,{ }^{* * *} \mathrm{P}<0.01$ and ${ }^{* * * *} \mathrm{P}<0.0001$ vs. the control group. ALT, alantolactone; ctrl, control.

ROS accumulation is associated with ALT-induced apoptosis. Several studies have revealed that anti-tumor drug-induced cell death is associated with intracellular ROS accumulation in multiple cancer types (26-28). Therefore, whether ROS were involved in ALT-induced apoptosis was also investigated. Intracellular ROS generation was detected following treatment with different ALT concentrations $(0,10,20,40$ or $60 \mu \mathrm{M})$ for $6 \mathrm{~h}$. ALT significantly increased intracellular ROS generation in a concentration-dependent manner in BGC-823 cells (data not shown). Subsequently, to determine whether the increased ROS generation served an important role in ALT-induced cell apoptosis, the cells were pretreated with NAC $1 \mathrm{~h}$ prior to treatment with ALT for $24 \mathrm{~h}$. Pretreatment with NAC effectively prevented ALT-induced ROS accumulation (Fig. 4A). Additionally, NAC also abolished ALT-inhibited cell proliferation and ALT-induced cell apoptosis (Fig. 4B and C). Furthermore, western blot analysis revealed that NAC decreased the expression of Bax and the cleavage of PARP, and increased Bcl-2 expression (Fig. 4D). Collectively, the results indicated that ALT-induced apoptosis was associated with ROS accumulation.

ALT induces BGC-823 cell apoptosis via inhibition of AKT signaling. Accumulating evidence has revealed that the AKT pathway is one of the major signaling pathways closely associated with cancer progression $(29,30)$. To determine whether the AKT signaling pathway is involved in ALT-induced apoptosis, western blot analysis was used to assay the effects of ALT on p-AKT and AKT protein expression. As presented in Fig. 5A, BGC-823 cells were treated with $40 \mu \mathrm{M}$ ALT for $3,6,12$ and $24 \mathrm{~h}$. The phosphorylation of AKT was significantly decreased in a time-dependent manner. ROS have been demonstrated to be associated with the apoptosis induced by anti-cancer drugs via regulation of AKT pathways (31). To additionally investigate the association between ROS and the AKT pathway, cells were treated with or without NAC for $1 \mathrm{~h}$, and then western blot analysis was used to examine p-AKT and AKT protein expression levels in BGC-823 cells. As presented in Fig. 5B, NAC significantly increased AKT phosphorylation. 


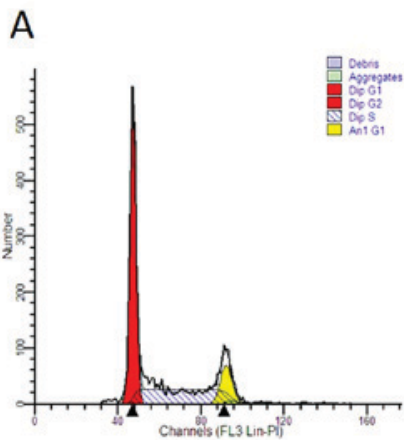

ctrl

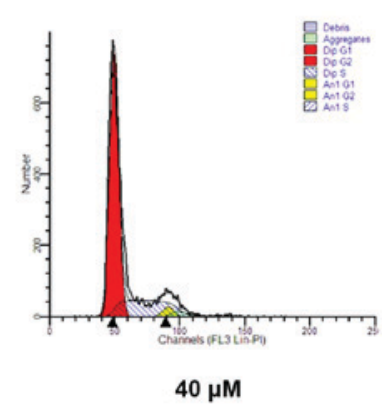

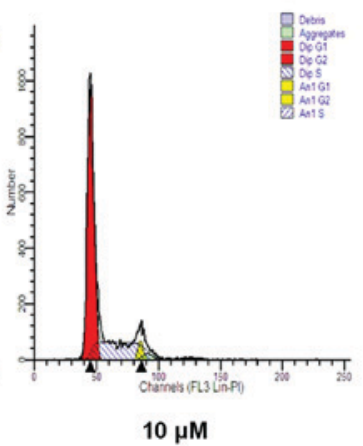

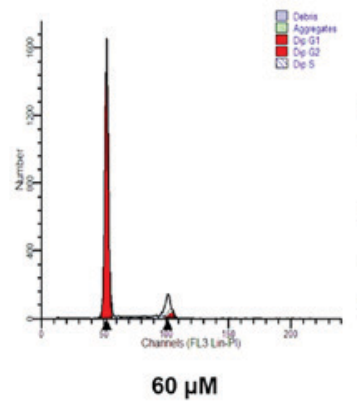

$60 \mu \mathrm{M}$

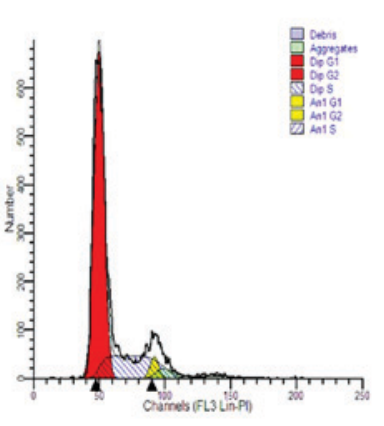

$20 \mu \mathrm{M}$

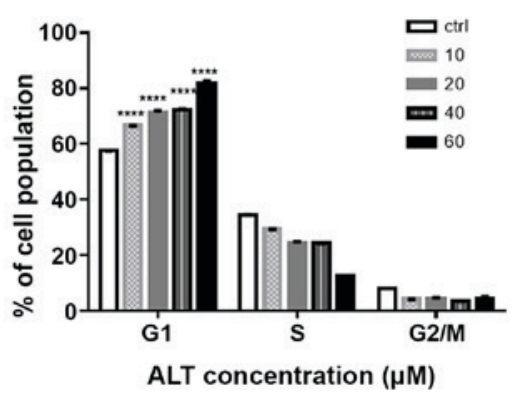

B

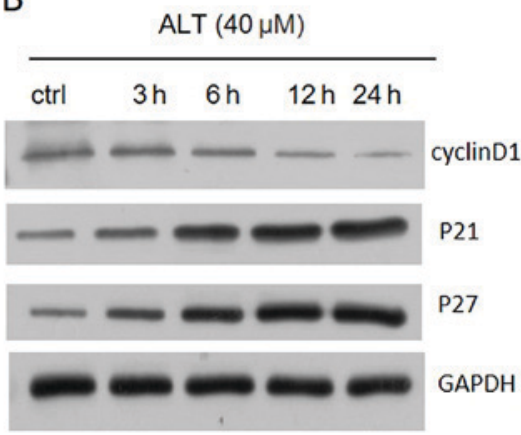

Figure 2. Effects of ALT on cell cycle distribution and cell cycle-associated proteins in BGC-823 cells. (A) BGC-823 cells were incubated with different concentrations of ALT $(0-60 \mu \mathrm{M})$ for $24 \mathrm{~h}$. The percentages of cells in G0/G1, S, and G2/M phases were determined by flow cytometry. All data are expressed as means \pm standard deviation from 3 independent experiments. (B) BGC-823 cells were treated with ALT (40 $\mu \mathrm{M})$ for 3, 6, 12 and $24 \mathrm{~h}$. Western blot analysis was used to detect the expression levels of cyclin D1, p21 and p27. GAPDH was used as an internal control. ${ }^{* * * *}$ P<0.0001 vs. the control group. ALT, ALT, alantolactone; ctrl, control; p21, cyclin-dependent protein kinase 1; p27, cyclin-dependent protein kinase 1B.

Together, these results suggest that ALT induced apoptosis through ROS-mediated inactivation of the AKT signaling pathway in BGC-823 cells.

\section{Discussion}

The emergence of drug resistance remains a major barrier for successful cancer treatments. Therefore, exploring and developing novel and effective anti-cancer drugs is essential. ALT, a sesquiterpene lactone, is a potential anti-cancer therapeutic agent. In a previous study, the anti-cancer effects of ALT were demonstrated in various tumor cells, including breast (14), lung (20,32), cervical $(17,18)$, liver (12), colorectal (10) and colon cancer (33). However, to the best of our knowledge, there is a lack of thorough experimental data describing the anti-tumor effects of ALT in BGC-823 human gastric cancer cells. The present study demonstrated that ALT exhibited significant anti-cancer effects in BGC-823 cells: ALT inhibited cell proliferation, and induced G0/G1 cell cycle arrest and apoptosis in BGC-823 cells. Furthermore, ALT promoted BGC-823 cell apoptosis via ROS-induced AKT signaling.

Cell cycle arrest is an important mechanism associated with anti-cancer drug-induced proliferation inhibition $(34,35)$. Previous studies have indicated that ALT may inhibit cell cycle progression in lung and colorectal cancer cells $(10,32)$. Similarly, flow cytometric analysis demonstrated that ALT induced $\mathrm{G} 0 / \mathrm{G} 1$ cell cycle arrest of BGC-823 cells in a concentration-dependent manner. Furthermore, previous studies have demonstrated that anti-cancer drugs promote G0-G1/S transition and inhibit cell proliferation by regulating the expression of cyclin D1 (36,37). Additionally, p27 and p21 serve suppressive roles in G0-G1/S transition by inhibiting the activity of cyclin/cyclin-dependent kinase complexes $(37,38)$. To additionally investigate the molecular basis by which ALT inhibited G0/G1 transition in BGC-823 cells, the expression of cell cycle-associated proteins was determined by 
A

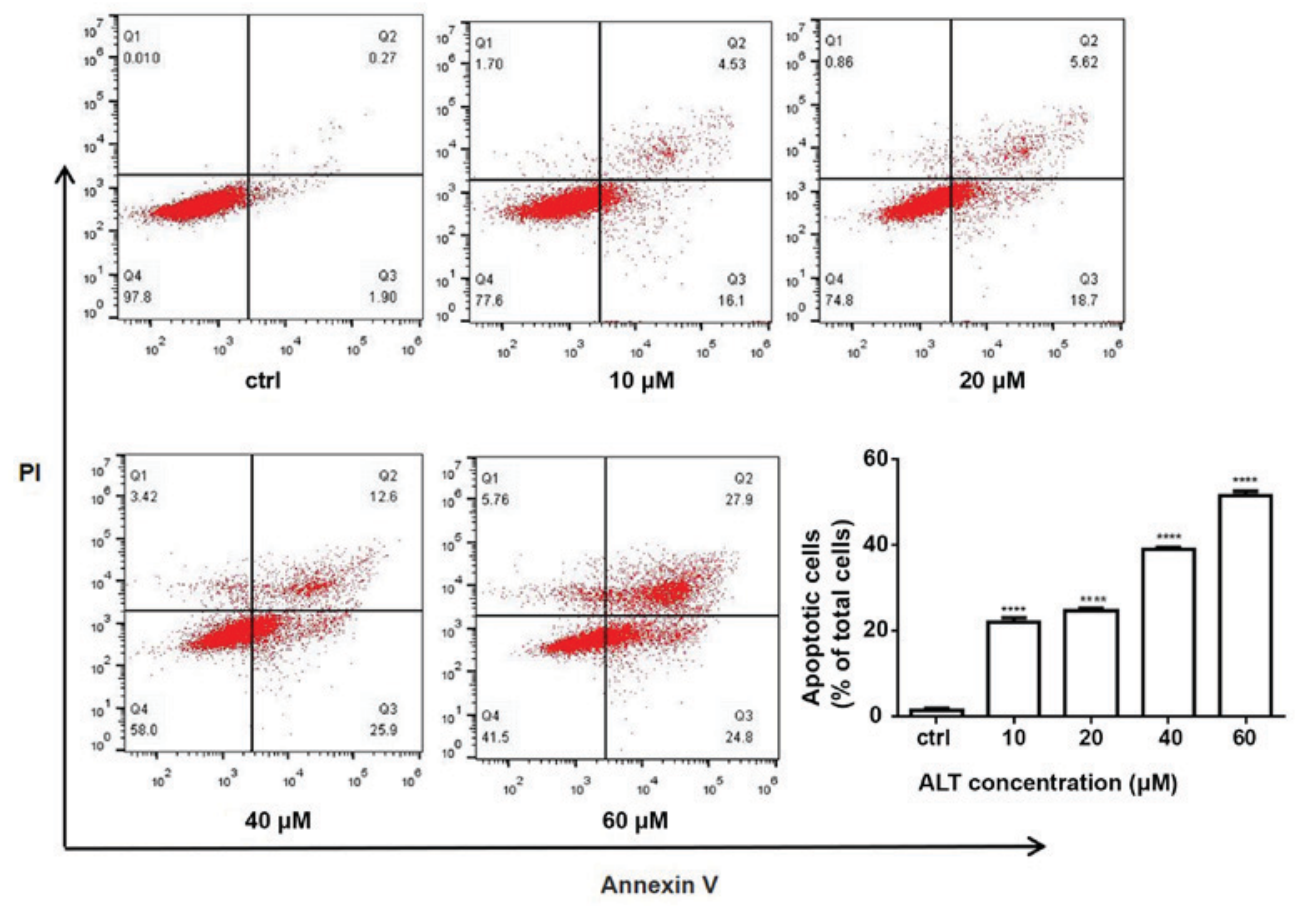

B

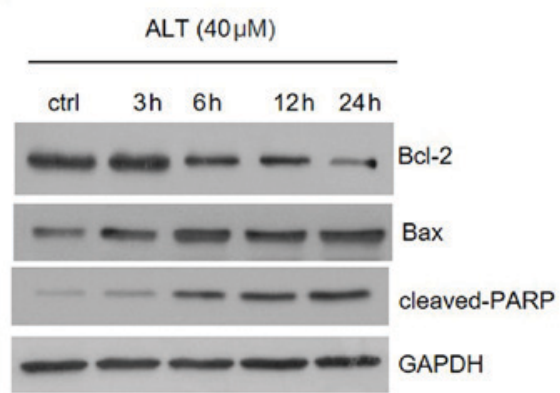

Figure 3. Effects of ALT on cell cycle distribution and cell apoptosis-associated proteins in BGC-823 cells. (A) BGC-823 cells were treated with the indicated concentrations of ALT $(0-60 \mu \mathrm{M})$ for $24 \mathrm{~h}$ and cell apoptosis was examined in BGC-823 cells by flow cytometry. (B) BGC-823 cells treated with ALT ( $40 \mu \mathrm{M})$ for 3, 6, 12 and $24 \mathrm{~h}$. Western blot analysis was used to detect the expression levels of Bax, Bcl-2 and cleaved PARP. GAPDH was used as an internal control. ${ }^{* * * * *} \mathrm{P}<0.0001$ vs. the control group. ALT, alantolactone; Bcl-2, B cell lymphoma 2; Bax, Bcl-2-associated protein; PARP, poly (adenosine 5'diphosphate-ribose) polymerase; PI, propidium iodide.

western blot analysis. ALT markedly decreased the expression levels of cyclin D1, whereas the expression of p21 and p27 were increased. These data provide evidence that ALT inhibited the proliferation of BGC- 823 cells via ALT-induced G0/G1 phase arrest.

Apoptosis is a critical homeostatic mechanism involved in anti-cancer drug-induced proliferation inhibition (35). The mitochondrial apoptotic pathway has a critical role in drug-mediated apoptosis and is often regarded as a potential anti-cancer target (39-41). Bcl-2 family protein members, including Bax and Bcl-2, serve important roles in the mitochondrial apoptotic pathway. Previous data has indicated that ALT induces apoptosis in a variety of human cancer cells: Cui et al (14) suggested that ALT induced the mitochondrial-mediated apoptotic pathway by increasing the $\mathrm{Bax} / \mathrm{Bcl}-2$ ratio and PARP cleavage in MDA-MB-231 cells; Jiang et al (17) also demonstrated that the Bcl-2/Bax signaling pathway was associated with ALT-induced HeLa cell apoptosis. In the present study, treatment with ATL significantly induced apoptosis of BGC-823 cells by increasing the $\mathrm{Bax} / \mathrm{Bcl}-2$ ratio and PARP cleavage in BGC-823 cells. These data suggest that the mitochondrial apoptotic pathway serves a key role in ALT-mediated BGC-823 cell apoptosis.

The AKT signaling pathway is an important anti-apoptosis pathway that promotes cell survival and resistance to cell apoptosis induced by chemotherapeutic agents in various cancer types (42). Inactivation of the AKT signaling pathway may inhibit cell growth and induce cell apoptosis in various cancer cells. Therefore, whether the AKT pathway was associated with ALT-induced apoptosis of BGC-823 cells was examined in the present study. The data demonstrated that ALT decreased the phosphorylation of AKT in a time-dependent manner. This indicated that AKT signaling may be involved in ALT-mediated apoptosis of BGC-823 cells.

ROS have been suggested to be involved in the initiation and the promotion of tumor development at different stages of carcinogenesis $(43,44)$. In fact, numerous studies have 

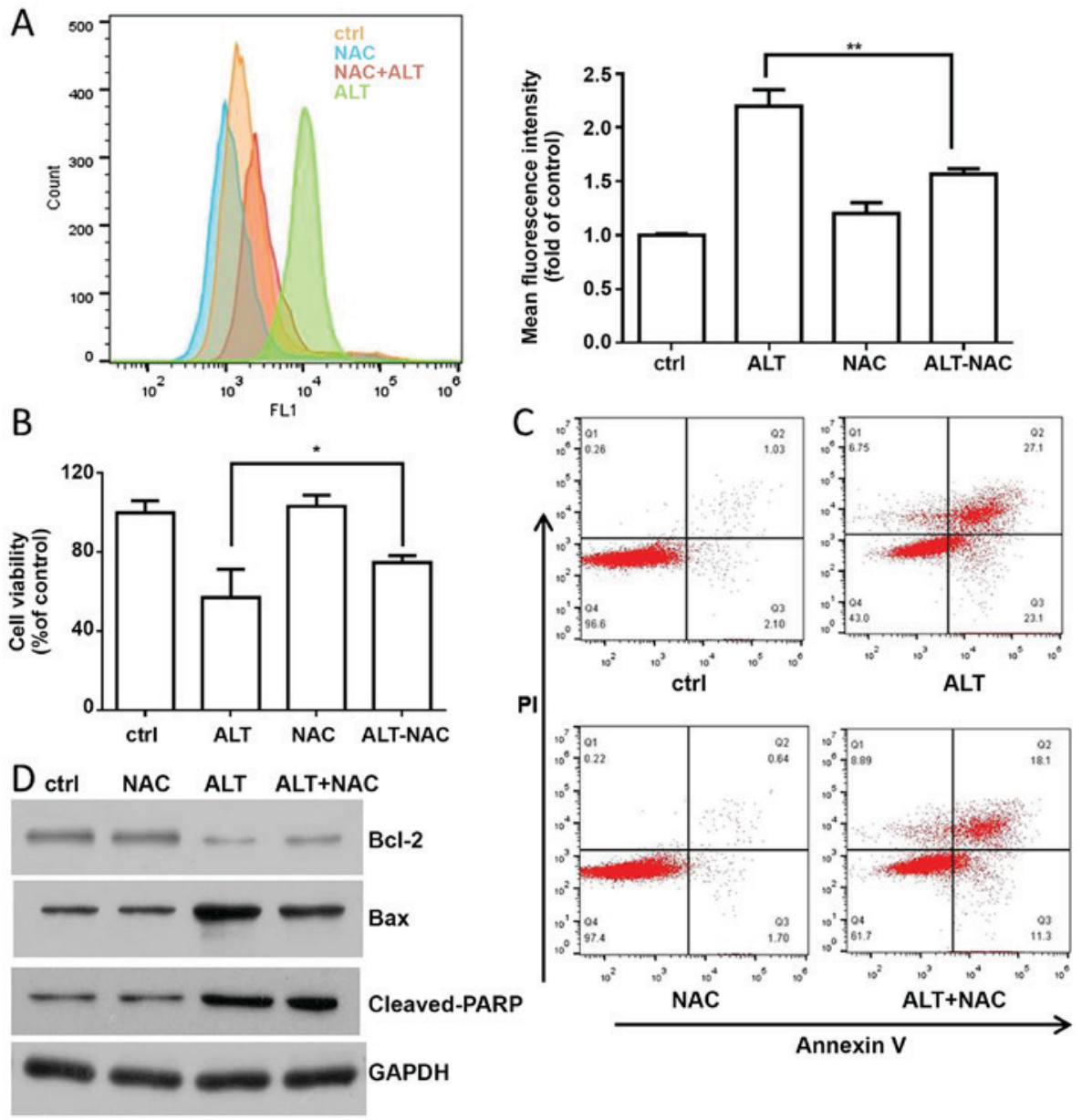

Annexin V

Figure 4. ROS are involved in ALT-induced apoptosis. (A) BGC-823 cells were pre-treated with NAC (10 mM) for $1 \mathrm{~h}$, and then ALT (40 $\mu \mathrm{M})$ was added to the cells for $6 \mathrm{~h}$. Flow cytometry was used to detect ROS levels. (B) BGC-823 cells were pre-treated with NAC (10 mM) for $1 \mathrm{~h}$, and then ALT (40 $\mu \mathrm{M})$ was added to the cells for $24 \mathrm{~h}$. MTT was used to measure cell viability. (C) BGC-823 cells were incubated with ALT and NAC as aforementioned. Flow cytometry was used to detect apoptosis in BGC-823 cells. (D) BGC-823 cells were pre-treated with NAC (10 mM) for $1 \mathrm{~h}$, and then ALT (40 $\mu \mathrm{M})$ was added to the cells for $12 \mathrm{~h}$. Western blot analysis was used to detect the expression levels of Bax, Bcl-2 and cleaved PARP. ${ }^{*} \mathrm{P}<0.05$ and ${ }^{* *} \mathrm{P}<0.01$ vs. the control group. ROS, reactive oxygen species; ALT, alantolactone; NAC, N-acetyl cysteine; Bcl-2, B cell lymphoma 2; Bax, Bcl-2-associated protein; PARP, poly (adenosine 5 'diphosphate-ribose) polymerase; PI, propidium iodide; ctrl, control.

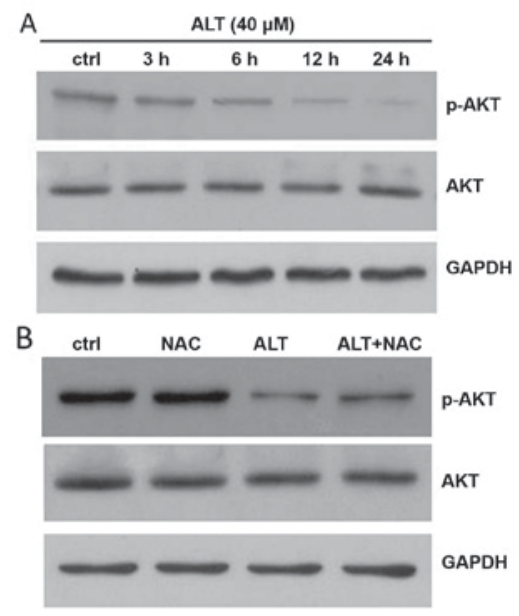

Figure 5. ALT induces BGC-823 cell apoptosis via reactive oxygen species-mediated AKT signaling. (A) BGC-823 cells were treated with ALT $(40 \mu \mathrm{M})$ for 3, 6, 12 and $24 \mathrm{~h}$. The levels of p-AKT and AKT were analyzed by western blot analysis. (B) BGC-823 cells were pre-incubated with NAC $(10 \mathrm{mM})$ for $1 \mathrm{~h}$, and then ALT $(40 \mu \mathrm{M})$ was added to the cells for $12 \mathrm{~h}$. The expression levels of p-AKT and AKT were measured by western blot analysis. ALT, alantolactone; NAC, N-acetyl cysteine; AKT, RAC-alpha serine/threonine-protein kinase; $p$, phosphorylated; ctrl, control. demonstrated that various anti-cancer drugs exert their effects via ROS-dependent pathways (45-47). ALT was demonstrated to induce apoptosis of MDA-MB-231 cells via ROS-mediated mitochondrial dysfunction (14). Jiang et al (17) also revealed that ROS may mediate apoptosis in human cervical cancer cells by increasing the $\mathrm{Bax} / \mathrm{Bcl}-2$ ratio. The results of the present study demonstrated that ALT treatment increased ROS generation in a concentration-dependent manner in BGC-823 cells (data not shown). Furthermore, pretreatment with NAC for $1 \mathrm{~h}$ reversed the ALT-induced production of ROS and cell apoptosis, and NAC significantly decreased the Bax/Bcl-2 ratio and PARP cleavage. The results suggested that ALT induced ROS-dependent apoptosis in BGC-823 cells. In addition, ROS generation was demonstrated to be involved in chemotherapeutic agent-mediated apoptosis and may be an upstream regulator of AKT-mediated signaling pathways $(48,49)$. In the present study, pretreatment with NAC for $1 \mathrm{~h}$ reversed the AKT inhibition induced by ALT. These results suggested that the apoptosis of BGC-823 cells was induced by ALT via ROS generation, which was then modulated the AKT signaling.

In conclusion, the results demonstrated that ALT induced apoptosis and G0/G1 phase arrest in BGC-823 cells in a 
concentration-dependent manner. In addition, ALT induced the apoptosis of BGC-823 cells via ROS-mediated inactivation of the AKT signaling pathway; therefore, ALT may be a promising candidate drug for the treatment of gastric cancer. However, additional studies are required to validate the anti-cancer activity of ALT in xenograft mouse models in vivo.

\section{Acknowledgements}

Not applicable.

\section{Funding}

The present study was supported by the Xinjiang Uygur Autonomous Region Natural Science Foundation Project (grant no. 2015211C244).

\section{Availability of data and materials}

The datasets used and/or analyzed in the present study are available from the corresponding author on reasonable request.

\section{Authors' contributions}

$\mathrm{XZ}$ conducted the experiments, analyzed the data, contributed to the design of the study and prepared the manuscript. XZ and HMZ performed the western blotting and analyzed the data. All authors read and approved the final manuscript.

\section{Ethics approval and consent to participate}

Not applicable.

\section{Patient consent for publication}

Not applicable.

\section{Competing interests}

The authors declare that they have no competing interests.

\section{References}

1. Ferlay J, Soerjomataram I, Dikshit R, Eser S, Mathers C, Rebelo M, Parkin DM, Forman D and Bray F: Cancer incidence and mortality worldwide: Sources, methods and major patterns in GLOBOCAN 2012. Int J Cancer 136: E359-E386, 2015.

2. Theuer CP, Kurosaki T, Ziogas A, Butler J and Anton-Culver H: Asian patients with gastric carcinoma in the United States exhibit unique clinical features and superior overall and cancer specific survival rates. Cancer 89: 1883-1892, 2000.

3. Lemjabbar-Alaoui H, Hassan OU, Yang YW and Buchanan P: Lung cancer: Biology and treatment options. Biochim Biophys Acta 1856: 189-210, 2015

4. Dikken JL, van de Velde CJ, Coit DG, Shah MA, Verheij M and Cats A: Treatment of resectable gastric cancer. Therap Adv Gastroenterol 5: 49-69,2012.

5. Ueno T, Iida M, Yoshino S, Takeda S, Kubota H, Higashida M, Oka Y, Tsuruta A, Matsumoto $\mathrm{H}$ and Nagano $\mathrm{H}$ : East versus west: Differences in surgical management in asia compared with Europe and North America. Surg Clin North Am 97: 453-466, 2017.
6. Kubatka P, Kapinova A, Kruzliak P, Kello M, Výbohová D, Kajo K, Novák M, Chripková M, Adamkov M, Péč M, et al: Antineoplastic effects of Chlorella pyrenoidosa in the breast cancer model. Nutrition 31: 560-569, 2015.

7. Pan J, Miao D and Chen L: Germacrone reverses adriamycin resistance in human chronic myelogenous leukemia K562/ADM cells by suppressing MDR1 gene/P-glycoprotein expression. Chem Biol Interact 288: 32-37, 2018.

8. Zhang X, Lan D, Ning S and Ruan L: Anticancer action of lactucopicrin in SKMEL-5 human skin cancer cells is mediated via apoptosis induction, G2/M cell cycle arrest and downregulation of $\mathrm{m}=$ TOR/PI3K/AKT signalling pathway. J BUON 23: 224-228, 2018.

9. Khan M, Yi F, Rasul A, Li T, Wang N, Gao H, Gao R and Ma T: Alantolactone induces apoptosis in glioblastoma cells via GSH depletion, ROS generation, and mitochondrial dysfunction. IUBMB Life 64: 783-794, 2012.

10. Ding Y, Wang H, Niu J, Luo M, Gou Y, Miao L, Zou Z and Cheng Y: Induction of ROS overload by alantolactone prompts oxidative DNA damage and apoptosis in colorectal cancer cells. Int J Mol Sci 17: 558, 2016.

11. Lei JC, Yu JQ, Yin Y, Liu YW and Zou GL: Alantolactone induces activation of apoptosis in human hepatoma cells. Food Chem Toxicol 50: 3313-3319, 2012.

12. Khan M, Li T, Ahmad Khan MK, Rasul A, Nawaz F, Sun M, Zheng Y and Ma T: Alantolactone induces apoptosis in HepG2 cells through GSH depletion, inhibition of STAT3 activation, and mitochondrial dysfunction. Biomed Res Int 2013: 719858, 2013.

13. Yang C, Yang J, Sun M, Yan J, Meng X and Ma T: Alantolactone inhibits growth of K562/adriamycin cells by downregulating $\mathrm{Bcr} / \mathrm{Abl}$ and P-glycoprotein expression. IUBMB Life 65: 435-444, 2013.

14. Cui L, Bu W, Song J, Feng L, Xu T, Liu D, Ding W, Wang J, Li C, $\mathrm{Ma}$ B, et al: Apoptosis induction by alantolactone in breast cancer MDA-MB-231 cells through reactive oxygen species-mediated mitochondrion-dependent pathway. Arch Pharm Res 41: 299-313, 2018.

15. Lee SS, Jeong HE, Liu KH, Ryu JY, Moon T, Yoon CN, Oh SJ, Yun $\mathrm{CH}$ and Shin JG: Identification and functional characterization of novel CYP2J2 variants: G312R variant causes loss of enzyme catalytic activity. Pharmacogenet Genomics 15: 105-113, 2005.

16. Gaedigk A, Baker DW, Totah RA, Gaedigk R, Pearce RE, Vyhlidal CA, Zeldin DC and Leeder JS: Variability of CYP2J2 expression in human fetal tissues. J Pharmacol Exp Ther 319: 523-532, 2006

17. Jiang Y, Xu H and Wang J: Alantolactone induces apoptosis of human cervical cancer cells via reactive oxygen species generation, glutathione depletion and inhibition of the $\mathrm{Bcl}-2 / \mathrm{Bax}$ signaling pathway. Oncol Lett 11: 4203-4207, 2016.

18. Zhang J, Li Y, Duan D, Yao J, Gao K and Fang J: Inhibition of thioredoxin reductase by alantolactone prompts oxidative stress-mediated apoptosis of HeLa cells. Biochem Pharmacol 102: 34-44, 2016.

19. Liu YR, Cai QY, Gao YG, Luan X, Guan YY, Lu Q, Sun P, Zhao $\mathrm{M}$ and Fang C: Alantolactone, a sesquiterpene lactone, inhibits breast cancer growth by antiangiogenic activity via blocking VEGFR2 signaling. Phytother Res 32: 643-650, 2018.

20. Maryam A, Mehmood T, Zhang H, Li Y, Khan M and Ma T: Alantolactone induces apoptosis, promotes STAT3 glutathionylation and enhances chemosensitivity of A549 lung adenocarcinoma cells to doxorubicin via oxidative stress. Sci Rep 7: 6242, 2017.

21. Yu ZY, Wang Z, Lee KY, Yuan P and Ding J: Effect of silencing colon cancer-associated transcript 2 on the proliferation, apoptosis and autophagy of gastric cancer BGC-823 cells. Oncol Lett 15: 3127-3132, 2018.

22. Wen W, Lowe G, Roberts CM, Finlay J, Han ES, Glackin CA and Dellinger TH: Pterostilbene Suppresses ovarian cancer growth via induction of apoptosis and blockade of cell cycle progression involving inhibition of the STAT3 pathway. Int J Mol Sci 19: pii: E1983, 2018

23. Ling Z, Guan H, You Z, Wang C, Hu L, Zhang L, Wang Y, Chen S, $\mathrm{Xu} \mathrm{B}$ and Chen M: Aloperine executes antitumor effects through the induction of apoptosis and cell cycle arrest in prostate cancer in vitro and in vivo. Onco Targets Ther 11: 2735-2743, 2018.

24. Cho HD, Lee JH, Moon KD, Park KH, Lee MK and Seo KI: Auriculasin-induced ROS causes prostate cancer cell death via induction of apoptosis. Food Chem Toxicol 111: 660-669, 2018.

25. Lai ZQ, Ip SP, Liao HJ, Lu Z, Xie JH, Su ZR, Chen YL, Xian YF, Leung PS and Lin ZX: Brucein D, a naturally occurring tetracyclic triterpene quassinoid, induces apoptosis in pancreatic cancer through ROS-associated PI3K/Akt signaling pathway. Front Pharmacol 8: 936, 2017. 
26. Xia S, Miao Y and Liu S: Withaferin A induces apoptosis by ROS-dependent mitochondrial dysfunction in human colorectal cancer cells. Biochem Biophys Res Commun 503: 2363-2369, 2018.

27. Xu Y, Tong Y, Ying J, Lei Z, Wan L, Zhu X, Ye F, Mao P, Wu X, Pan R, et al: Chrysin induces cell growth arrest, apoptosis, and ER stress and inhibits the activation of STAT3 through the generation of ROS in bladder cancer cells. Oncol Lett 15: 9117-9125, 2018.

28. Sun Q, Lu NN and Feng L: Apigetrin inhibits gastric cancer progression through inducing apoptosis and regulating ROS-modulated STAT3/JAK2 pathway. Biochem Biophys Res Commun 498: 164-170, 2018.

29. Sathe A and Nawroth R: Targeting the PI3K/AKT/mTOR Pathway in Bladder Cancer. Methods Mol Biol 1655: 335-350, 2018.

30. Costa RLB, Han HS and Gradishar WJ: Targeting the PI3K/AKT/mTOR pathway in triple-negative breast cancer: A review. Breast Cancer Res Treat 169: 397-406, 2018.

31. Wang B, Zhou TY, Nie CH, Wan DL and Zheng SS: Bigelovin, a sesquiterpene lactone, suppresses tumor growth through inducing apoptosis and autophagy via the inhibition of mTOR pathway regulated by ROS generation in liver cancer. Biochem Biophys Res Commun 499: 156-163, 2018.

32. Zhao P, Pan Z, Luo Y, Zhang L, Li X, Zhang G, Zhang Y, Cui R, Sun M and Zhang X: Alantolactone induces apoptosis and cell cycle arrest on lung squamous cancer SK-MES-1 cells J Biochem Mol Toxicol 29: 199-206, 2015.

33. Zhang Y, Bao YL, Wu Y, Yu CL, Huang YX, Sun Y, Zheng LH and $\mathrm{Li}$ YX: Alantolactone induces apoptosis in RKO cells through the generation of reactive oxygen species and the mitochondrial pathway. Mol Med Rep 8: 967-972, 2013.

34. King KL and Cidlowski JA: Cell cycle regulation and apoptosis. Annu Rev Physiol 60: 601-617, 1998.

35. Sithara T, Dhanya BP, Arun KB, SiniS, Dan M,Kokkuvayil Vasu R and Nisha P: Zerumbone, a cyclic sesquiterpene from Zingiber zerumbet induces apoptosis, cell cycle arrest, and antimigratory effects in SW480 colorectal cancer cells. J Agric Food Chem 66: 602-612, 2018

36. Wang J, Li XM, Bai Z, Chi BX, Wei Y and Chen X: Curcumol induces cell cycle arrest in colon cancer cells via reactive oxygen species and Akt/GSK3 $\beta /$ cyclin D1 pathway. J Ethnopharmacol 210: 1-9, 2018.

37. Sikander M, Hafeez BB, Malik S, Alsayari A, Halaweish FT, Yallapu MM, Chauhan SC and Jaggi M: Cucurbitacin D exhibits potent anti-cancer activity in cervical cancer. Sci Rep 6: 36594, 2016.

38. Cicenas J, Kalyan K, Sorokinas A, Jatulyte A, Valiunas D, Kaupinis A and Valius M: Highlights of the latest advances in research on CDK inhibitors. Cancers (Basel) 6: 2224-2242, 2014.
39. Sithara T, Arun KB, Syama HP, Reshmitha TR and Nisha P: Morin inhibits proliferation of SW480 colorectal cancer cells by inducing apoptosis mediated by reactive oxygen species formation and uncoupling of warburg effect. Front Pharmacol 8: 640, 2017.

40. Choi AY, Choi JH, Yoon H, Hwang KY, Noh MH, Choe W, Yoon KS, Ha J, Yeo EJ and Kang I: Luteolin induces apoptosis through endoplasmic reticulum stress and mitochondrial dysfunction in Neuro-2a mouse neuroblastoma cells. Eur J Pharmacol 668: 115-126, 2011.

41. Shahali A, Ghanadian M,Jafari SM and Aghaei M: Mitochondrial and caspase pathways are involved in the induction of apoptosis by nardosinen in MCF-7 breast cancer cell line. Res Pharm Sci 13: 12-21, 2018.

42. Park J, Ko YS, Yoon J, Kim MA, Park JW, Kim WH, Choi Y, Kim JH, Cheon Y and Lee BL: The forkhead transcription factor FOXO1 mediates cisplatin resistance in gastric cancer cells by activating phosphoinositide 3-kinase/Akt pathway. Gastric Cancer 17: 423-430, 2014.

43. Zheng QS and Zheng RL: Effects of ascorbic acid and sodium selenite on growth and redifferentiation in human hepatoma cells and its mechanisms. Pharmazie 57: 265-269, 2002.

44. Balaban RS, Nemoto S and Finkel T: Mitochondria, oxidants, and aging. Cell 120: 483-495, 2005.

45. Chetram MA,Bethea DA,Odero-Marah VA,Don-Salu-Hewage AS, Jones KJ and Hinton CV: ROS-mediated activation of AKT induces apoptosis via pVHL in prostate cancer cells. Mol Cell Biochem 376: 63-71, 2013.

46. Pavithra PS, Mehta A and Verma RS: Aromadendrene oxide 2 , induces apoptosis in skin epidermoid cancer cells through ROS mediated mitochondrial pathway. Life Sci 197: 19-29, 2018.

47. Cui YQ, Liu YJ and Zhang F: The suppressive effects of Britannin (Bri) on human liver cancer through inducing apoptosis and autophagy via AMPK activation regulated by ROS. Biochem Biophys Res Commun 497: 916-923, 2018.

48. Zhong WF, Wang XH, Pan B, Li F, Kuang L and Su ZX: Eupatilin induces human renal cancer cell apoptosis via ROS-mediated MAPK and PI3K/AKT signaling pathways. Oncol Lett 12: 2894-2899, 2016.

49. Xu H, Li X, Ding W, Zeng X, Kong H, Wang H and Xie W: Deguelin induces the apoptosis of lung cancer cells through regulating a ROS driven Akt pathway. Cancer Cell Int 15: 25, 2015.

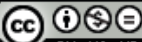

This work is licensed under a Creative Commons Attribution-NonCommercial-NoDerivatives 4.0 International (CC BY-NC-ND 4.0) License. 